\title{
Hématome sous dural bilatéral après analgesie péridurale
}

Pierre Diemunsch $M D,{ }^{*}$ Valérie Pichon Balabaud MD, ${ }^{*}$ Christophe Petiau MD, ${ }^{\dagger}$ Christian Marescaux $\mathrm{MD},{ }^{\dagger}$ André Muller MD, ${ }^{*}$ Jocelyne Valfrey $\mathrm{MD},{ }^{*}$ Alain Treisser $M D^{*}$

Purpose: The authors report a case of bilateral subdural hematoma (SDH) which occurred following epidural analgesia for labour, complicated by post dural puncture headache (PDPH). Physiopathological mechanisms are discussed.

Clinical features: A 27-yr-old woman displayed typical PDPH following epidural anaesthesia. On the fifth day she was given a blood patch (BP) which proved immediately effective. Further developments were marked by late recurrence of PDPH and by administration of a second BP on the 24th day. With the aggravation of the headaches, the disapearance of their postural nature and with the appearance of transitory focal neurological signs on the 30th day, a CT-Scan was done and showed bilateral subdural haematoma. Following surgical drainage, the patient made an uneventful recovery.

Conclusion: The presence of PDPH complicated by a typical neurological deterioration should prompt the anaesthetist to seek an immediate clinical and $x$-ray diagnosis in order to look for the existence of intracranial complications.

Objectif: Les auteurs rapportent le cas d'un hématome sous dural (HSD) bilatéral survenu au décours d'une analgésie péridurale pour l'accouchement, compliquée de céphalées post effraction de la dure-mère. Plusieurs mécanismes physiopathologiques sont envisagés.

Eléments cliniques: Une femme de 27 ans présentait des céphalées typiques dans les suites d'une analgésie péridurale obstétricale. Le $5^{\mathrm{e}}$ jour après l'accouchement, elle bénéficiait d'un pansement de sang épidural (blood patch, BP) immédiatement efficace. L'évolution était marquée par la récidive tardive de céphalées et par la réalisation d'un second BP au 24e jour. Devant l'aggravation des céphalées, la disparition de leur caractère postural et l'apparition de signes neurologiques focaux transitoires le $30^{\circ}$ jour, on découvrait par tomodensitométrie, l'existence d'un hématome sous dural bilatéral. L'évolution fût favorable sans séquelle après son évacuation chirurgicale.

Conclusion: La présence de céphalées post effraction dureméro-arachnoïdienne compliquées de signes neurologiques même discrets et transitoires doit conduire l'anesthésiste à demander un diagnostic neurologique clinique et radiologique immédiat afin de rechercher et de traiter une éventuelle complication intracrânienne.

\footnotetext{
"Département d'Anesthésie GEPAR, †Clinique Neurologique, Hópitaux Universitaires de Strasbourg, 67000 Strasbourg, France. Adresser la correspondance: Pierre Diemunsch, Département d'Anesthésie GEPAR, Hôpitaux Universitaires de Strasbourg, 1, place de l'Hópital, 67000 Strasbourg, France.

Accepted for publication January 15, 1998.
} 


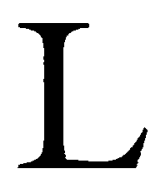

'EFFRACTION dureméro-arachnoïdienne (EDM) complique 0.26 à $2.6 \%$ des anesthésies péridurales (APD). Elle peut être la cause de céphalées typiques dites céphalées post effraction de la dure-mère (CPEDM). Lorsque une CPEDM devient persistante, rebelle aux traitements établis et surtout si elle perd ses caractères séméiologiques pathognomoniques et s'accompagne de signes. neurologiques, il faut évoquer la possibilité d'une complication intracrânienne et organiser son diagnostic et son éventuel traitement en urgence.

Nous rapportons le cas d'un hématome sous dural (HSD) bilatéral découvert quatre semaines après la réalisation d'une APD.

\section{Cas clinique}

Une primigeste de 27 ans pesant $57 \mathrm{~kg}$, était admise en maternité à 40 semaines d'aménorrhée pour un début de travail. Ses antécédents significatifs étaient limités à une appendicectomie sous anesthésie générale à 32 semaines de la présente grossesse. Le bilan biologique (numération formule sanguine, taux de plaquettes, coagulation, ionogramme sanguin) était normal.

A $5 \mathrm{~cm}$ de dilatation, une APD était mise en oeuvre par ponction de l'espace L2-L3 (kit Minipack type Portex Système $1^{\star}$; aiguille de Tuohy 18 gauge; technique du mandrin gazeux). Après aspiration et administration d'une dose test (lidocaïne 1.5\%: $45 \mathrm{mg}$, adrénaline: $1 / 160000$ ), $10 \mathrm{ml}$ de bupivacaïne $0.25 \%$ plus $25 \mathrm{mcg}$ de sufentanil étaient injectés par l'aiguille en deux fractions de $5 \mathrm{ml}$. L'insertion du cathéter (repère $8 \mathrm{~cm}$ à la peau) provoquait ensuite des épisodes de paresthésies fugaces. La patiente accouchait normalement d'un garçon de $3380 \mathrm{~g}$ après deux heurcs quarante minutes d'analgésie satisfaisante sans ajout d'anesthésiques et sans incident.

Les suites de couches furent marquées par une infection urinaire à streptocoque $B$ et par l'apparition dès le lendemain de l'accouchement (J+1) de CPEDM orthostatiques typiques sans autre signe neurologique. Malgré une amélioration initiale sous traitement conservateur (paracétamol $3 \times 500 \mathrm{mg} / \mathrm{j}$, décubitus, hydratation à $2000 \mathrm{ml} / \mathrm{j}$, caféine $2 \times 50 \mathrm{mg} / \mathrm{j}$ par voie intraveineuse), la persistance des céphalées faisait pratiquer un pansement de sang épidural (blood patch, BP) à J+5 (espace L3-L4; aiguille de Tuohy gauge 18; $10 \mathrm{ml}$ de sang autologue; décubitus dorsal strict maintenu 60 minutes après l'injection) permettant la sortie du service à $J+6$, tout symptôme ayant disparu.

A $J+23$, la patiente reprenait contact avec la maternité et signalait la réapparition depuis quelques jours de céphalées fronto-occipitales analogues aux CPEDM ressenties initialement mais sans leur caractère postural.
Elle fût alors immédiatement orientée vers un service de neurologie. L'examen d'admission ne révéla pas de syndrome particulier et une récidive tardive de CPEDM avec échec secondaire du BP fût le diagnostique retenu. Un second BP était réalisé le lendemain permettant un soulagement partiel des douleurs et le retour de la patiente à son domicile. $\mathrm{A} \mathrm{J+30}$ apparurent un épisode de paresthésies dans l'hémicorps gauche durant dix minutes et deux épisodes de paraphasie avec manque du mot. Les céphalées devenues bifrontales, constantes, non posturales, étaient accompagnées de sensations vertigineuses aux stations debout et assise. Un examen tomodensitométrique (TDM) avec injection de produit de contraste objectivait l'existence d'un HSD bilatéral avec compression du ventricule latéral gauche. La patiente était rapidement transférée dans un service de neurochirurgie pour évacuation des deux HSD $(60 \mathrm{ml}$ à gauche et $80 \mathrm{ml}$ à droite). L'évolution était favorable et la sortic autorisée le $6^{\circ}$ jour post opératoire $(J+36)$ sans aucune séquelle.

Un TDM de contrôle à 3 mois confirmait la disparition complète des hématomes, tandis que la patiente demeurait asymptomatique.

\section{Discussion}

Les CPEDM compliquent $86 \%$ des EDM accidentelles en obstétrique. ${ }^{1}$ Toutes les céphalées du post partum ne sont pas des CPEDM et 7\% des femmes n'ayant pas bénéficié d'une péridurale en présentent après leur accouchement.

Le plus souvent, l'EDM est flagrante, survient au cours de la recherche de l'espace péridural et se traduit par un reflux franc de liquide céphalo-rachidien (LCR). Ce reflux peut être absent spontanément et n'être révélé que par l'aspiration sur l'aiguille de Tuohy. Le test d'aspiration peut être lui même faussement négatif et l'EDM n'être finalement révélée qu'après l'administration de la dose test ou du premier bolus de la solution anesthésique. L'EDM peut également être le fait du cathéter pour lequel les précautions d'aspiration et de fractionnement des doses sont identiques à celles préconisées pour l'aiguille de Tuohy.

Dans d'autres cas, la brèche duremérienne n'est pas reconnue au cours de l'APD mais l'apparition de CPEDM typiques les jours suivants fait évoquer une effraction par le cathéter au moment de son insertion, ou plus tard, au cours du travail. L'effraction demeure occulte si aucune injection n'est pratiquée par ce cathéter. Cette explication est plausible chez notre patiente: le cathéter n'a pas servi et son insertion fût accompagnée de paresthésies.

Les CPEDM possèdent des caractères séméiologiques très évocateurs dont le plus spécifique est cer- 
tainement leur nature posturale. Elles apparaissent le plus souvent lors du premier lever, ou au cours des trois premiers jours, avec une localisation fronto-occipitale bilatérale et leur durée moyenne est de huit jours. ${ }^{2}$ Parfois suffisamment intenses pour empêcher la patiente de manger assise, elles sont volontiers associées à des nausées, vomissements, raideurs cervicales, sensations vertigineuses, acouphènes et troubles visuels.

Le mécanisme physiopathologique évoqué pour expliquer les céphalées serait la fuite de LCR à travers la brèche vers l'espace péridural. Cette fuite provoque d'une part le déplacement rostrocaudal du névraxe entraînant une tension sur les structures méningées crâniennes et d'autre part une vasodilatation cérébrale consécutive à la réduction du volume du LCR intracrânien..$^{3,5,7}$

Le traitement conservateur des CPEDM comprend: la prescription d'antalgiques, l'hyperhydratation (environ $2 \mathrm{l} / \mathrm{j}$ ) associée à un repos au lit et à l'administration de caféine (IV ou per os). Le traitement de référence des CPEDM rebelles au traitement conservateur est le BP. Le BP à but prophylactique demeure controversé en raison des risques d'EDM récurrente et de détérioration neurologique aiguë. ${ }^{9}$

L'HSD est une complication rare de l'APD, survenant après un délai moyen de 25 jours ( 30 heures à 5 mois) ${ }^{3,7}$ Ce diagnostic doit être pris en considération chez tout patient dont les céphalées persistent plus d'une semaine et perdent leur caractère postural, ou récidivent après une disparition initiale.,7 Les céphalées de l'HSD sont fréquemment associées à des troubles sensitivo-moteurs transitoires ( $90 \%$ des cas). On retrouve généralement une aphasie ( $77 \%$ des cas), des symptômes sensoriels (57\%), une hémiparésie $(50 \%)$ et des troubles visuels ( $3 \%$ des cas). La taille de l'hématome est mal corrélée à l'importance des symptômes observés. ${ }^{8}$ L'HSD s'accompagne de variations de perfusion intracérébrale aboutissant à des épisodes d'ischémie au pourtour de l'hématome et à des manifestations cliniques d'épilepsie ou d'aćcident ischémique cérébral transitoire (ICT). ${ }^{9}$ Les symptômes observés chez notre patiente sont compatibles avec ce cadre.

Parmi les facteurs de risque d'HSD au décours d'une EDM, on retrouve:

- l'âge avancé (par atrophie cérébrale et vulnérabilité de la paroi veineuse) ${ }^{7}$

- la déshydratation (responsable d'un collapsus des ventricules cérébraux $)^{4}$

- les pathologies intracrâniennes dont les tumeurs vascularisées du SNC et les antécédents de traumatisme crânien ${ }^{3,9}$
- les troubles de l'hémostase: coagulopathies et traitements anticoagulants (notamment héparine de bas poids moléculaire à but prophylactique $)^{10}$

- les antécédents d'EDM

- le nombre de tentatives effectuées lors de la ponction.

Chez la parturiente les facteurs prédisposant à la survenue d'un HSD sont représentés par l'augmentation des pressions dans les veines corticales lors des efforts de toux, de défécation, de poussée et par la fuite de LCR en cas d'EDM. ${ }^{4}$

Le diagnostic d'un HSD survenant secondairement après des céphalées n'est pas toujours facile à établir et il convient de le distinguer des autres complications possibles. Notamment la thrombophlébite, la méningite, l'hémorragie intracrânienne et l'ICT. ${ }^{8}$ En outre devant tout syndrome neurologique atypique survenant au décours d'une CPEDM, une TDM ou un examen par résonnance magnétique (IRM) s'imposent. La TDM doit impérativement s'accompagner d'une injection de produit de contraste pour éviter l'écueil de la phase isodense d'un HSD. ${ }^{3,11}$ Après tout traitement évacuateur d'un HSD, une réaccumulation de sang est possible. Sa fréquence pourrait atteindre $50 \%$ des cas. ${ }^{3}$ Un contrôle TDM ou IRM à 3 mois est donc souhaitable.

\section{Conclusion}

Dans le cas rapporté la combinaison de trois hypothèses étiopathogéniques peut être considérée pour expliquer l'HSD après une APD:

- Une brèche initiale par le cathéter passée inaperçue, aboutissant à la formation lente d'un HSD bilatéral.

- La réouverture d'une brèche après sa fermeture initiale par le premier BP.

- La formation d'une nouvelle brèche au cours de l'un des BP.

Nous retiendrons la possibilité d'une brèche duremérienne par le cathéter et d'une récidive de CPEDM par réouverture de cette brèche après un $\mathrm{BP}$ initialement satisfaisant. La reprise de la fuite du LCR pourrait être à l'origine des HSD.

Cette observation rappelle également que le BP peut avoir des échecs secondaires. L'évolution finalement favorable souligne l'absolue nécessité de la prise en charge spécialisée des détériorations neurologiques compliquant les CPEDM.

\section{References}

1 Stride PC, Cooper GM. Dural taps revisited. A 20 year survey from Birmingham maternity hospital. Anaesthesia 1993; 48: 247-55. 
2 Costignan SN, Sprigge JS. Dural puncture: the patient's perspective. Acta Anaesthesiol Scand 1996; 40: 710-4.

3 Ovanezian $G$, Rotbman A, Nazareno $G$, et al. Subdural hematoma after lumbar puncture. Report of 2 cases.

Am J Anesthesiol 1996; 23: 243-5, 252.

4 Deglaire $R$, Duverger P, Muckensturm $B$, et al. Hématome sous dural aigü intra cranien après une brèche dure mérienne accidentelle lors d'une anesthésie péridurale. Ann Fr Anesth Réanim 1988; 7: 156-8.

5 Eledjam J], Viel E, Aya $G$, et al. Les céphalées après ponction dure mérienne. Cah Anesthesiol 1993; 41: 579-88.

6 Cooper GM, Stride PC. Dural puncture. Blood patch not always benign. BMJ 1993; 306: 1339.

7 Vos PE, De Boer WA, Wurzer JAL, et al. Subdural hematoma after lumbar puncture: two cases reports and review of the literature. Clin Neurol Neurosurg 1991; 93: 127-32.

8 Kaminski HJ, Hlavin ML, Likavec MJ, et al. Transient neurologic deficit caused by chronic subdural hematoma. Am J Medicine 1992; 92: 698-700.

9 Beers $R A$, Cambareri JJ, Rodziewicz GS. Acute deterioration of mental status following epidural blood patch. Anesth Analg 1993; 76: 1147-9.

10 Wulf $H$. Epidural anaesthesia and spinal haematoma. Can J Anaesth 1996; 43: 1260-71.

11 Wyble SW, Baybi $D$, Webre $D$, et al. Bilateral subdural hematomas after dural puncture: delayed diagnosis after false negative computed tomography scan without contrast. Reg Anesth 1992; 17: 52-3. 